Old Pathway The traditional stress/rest MPS pathway requires two imaging sessions either the same day or on two separate days according to the patient's Body Mass Index (BMI). For a 1 day protocol typically the injected activity is split in a $1: 3$ ratio between the first and second injections. For BMI $<30$, a total of $1125 \mathrm{MBq}$ is administered. For BMI 30-35, a total of $1475 \mathrm{MBq}$ is administered. For $\mathrm{BMI}>35$, a total of 2000 $\mathrm{MBq}$ is administered $1: 1$. If the BMI is not provided by the requesting clinician then a letter is sent to the patient asking their height and weight.

New Pathway From September 2014 through December 2014, we introduced selective stress only imaging. A single high dose MIBI stress dose of of $800 \mathrm{MBq}$, was authorised for all patients, regardless of BMI. After stress imaging, a decision was made regarding the necessity of rest imaging.

Results Patients were referred from 10 hospitals. 407 patients were scanned. $55 \%$ were male and $45 \%$ female. Their average age was 66 years $(+/-12.9)$. Their average BMI was $29.4 \mathrm{~kg} / \mathrm{m}^{2}(+/-$ 6.4).

Of the total, $35 \%$ percent were normal at stress imaging and did not have rest imaging. Of the $65 \%$ who were abnormal, $60 \%$ of the combined rest/stress scans were interpreted as abnormal. As a result of this, the average time from request to appointment date for the stress scan reduced from 27 to 14 days.

The average time between stress scan and rest scan was 2.5 (range: 1-21 days). The average report turn around time from request to report for MPS was 5 days, but $97 \%$ of normal stress only scans were reported the same day. Furthermore, the administered radiation dose was lower for $67 \%$ of all patients.

In additional to scanning parameters being assessed, patients preferences were also assessed via a questionnaire. $49 \%$ expressed a preference for the test to be split across 2 days. $7 \%$ said either 1 day or 2 days. $20 \%$ of patients had to take a day off work to attend the hospital.

Conclusion Stress only MPS reduces radiation exposure, increases efficiency and thereby reduces cost.

\section{THE DEVELOPMENT OF A DIETARY PREPARATION PROTOCOL FOR OPTIMAL INFLAMMATORY IMAGING USING 18F-FDG PET}

${ }^{1}$ Eleanor Wicks*, ${ }^{2}$ Leon Menezes, ${ }^{2}$ Shane Blanchflower, ${ }^{2}$ Anna Barnes, ${ }^{2}$ Ashley Groves, ${ }^{3}$ Perry Elliott. ${ }^{1}$ University College London; ${ }^{2}$ UCLH; ${ }^{3}$ Barts Heat Centre; ${ }^{*}$ Presenting Author

\subsection{6/heartjnl-2016-309890.123}

Background Positron emission tomography (PET) performed utilising the glucose analogue and radiotracer 18F-FDG (FDG) to detect active inflammation, offers enormous potential for detecting disease presence and activity in myocarditis. However, the clinical utility of PET imaging is limited by the unpredictable nature of physiological FDG uptake within cardiac muscle. Methods to minimise physiological FDG uptake are necessary to depict active inflammation and to avoid avoid false negative and positive findings. Studies have suggested the use of fasting conditions to shift myocardial metabolism to primary free fatty acid (FFA) utilisation for energy and oxygen consumption to suppress physiological FDG uptake by normal myocardium. Specific dietary preparation regimens using a very high-fat content and low-carbohydrate diet prior to scanning have also been proposed, as has the administration of heparin to reduce FDG uptake through the activation of lipoprotein lipase (LPL) and hepatic lipase (HL) which enhances plasma lipolytic activity and leads to preferential free fatty acid consumption.

This study was therefore performed to develop an optimal dietary preparation protocol for imaging in PET/CT. We sought to establish whether patient preparation using heparin in addition to a high fat, low carbohydrate diet and prolonged fast prior to scanning ncreases the diagnostic capability of PET scanning in myocarditis.

Methods All patients referred for PET scans for the assessment of myocarditis were enrolled. We prospectively examined three preparation rationales to determine the optimal rationale for minimising physiological FDG-uptake. All subjects underwent strict preparation prior to scanning using one of the following protocols to enable comparison:

1. A 6-hour fast only, no dietary preparation.

2. Dietary preparation for $24 \mathrm{~h}$ prior to the scan with a high-fat content, low carbohydrate diet, followed by a prolonged fast for $12 \mathrm{~h}$.

3. Dietary preparation for $24 \mathrm{~h}$ prior to the scan with a high-fat content, low carbohydrate diet, followed by a prolonged fast for $12 \mathrm{~h}$ and the administration of $50 \mathrm{IU} / \mathrm{kg}$ of heparin prior to scanning.

The imaging findings were analysed by two independent and blinded clinicians. FDG uptake was categorised as none (complete suppression, no uptake $=0$ ), focal (localised uptake, $=1$ ), focal on diffuse (2) and diffuse (poor, failed suppression, $=4$ ).

Results A total of 280 PET scans were performed. 36 patients underwent preparation using a 6-hour fast only, 128 underwent dietary preparation and a 12-hour fast, and 118 underwent dietary preparation followed by a 12-hour fast and the administration of heparin prior to scanning.

A 6-hour fast alone was associated with a $44 \%$ failure to suppress physiological metabolism - equivalent to almost one in every two scans being suboptimal or un-interpretable. In contrast, restriction of carbohydrate intake with high fat intake alongside a prolonged 12-hour fast was successful in supressing normal myocardial metabolism in $89 \%$ of subjects. Similarly, of those who also received heparin, there was a failure to suppress normal myocardial metabolism in $11 \%$ of subjects. Conclusion Strict patient preparation prior to PET scanning is key to ensuring an optimal diagnostic disease assessment in myocarditis. A short duration of fasting of 6-hours or less alone is suboptimal in suppressing physiological uptake. In contrast, strict dietary preparation (using a high fat, low carbohydrate diet) and prolonged fast of $>12 \mathrm{~h}$ increases the diagnostic capability by $75 \%$. Interestingly, the addition of heparin to this regimen provided no additive benefit suggesting that the addition of heparin adds little to the diagnostic quality of scans and is therefore unnecessary.

\section{Valve disease/pericardial disease/ cardiomyopathy}

\section{THE USE OF NEXT GENERATION SEQUENCING TO DETERMINE GENOTYPE-PHENOTYPE CORRELATIONS IN DILATED CARDIOMYOPATHY}

${ }^{1}$ Eleanor Wicks*, ${ }^{2}$ Andrew Proven, ${ }^{3}$ Petros Syrris, ${ }^{3}$ Perry Elliott. ${ }^{1}$ University College London; ${ }^{2} \mathrm{UCLH}_{;}{ }^{3} \mathrm{UCL}$; ${ }^{*}$ Presenting Author

10.1136/heartjnl-2016-309890.124 